\title{
Article \\ Surviving Elderly Patients with Head-and-Neck Squamous Cell Carcinoma-What Is the Long-Term Quality of Life after Curative Radiotherapy?
}

\author{
Alexander Rühle ${ }^{1,2} \mathbb{D}^{\mathbb{D}}$, Erik Haehl ${ }^{1,2}$, Tobias Kalckreuth ${ }^{1,2}$, Raluca Stoian ${ }^{1,2} \mathbb{D}_{\text {, Simon K. B. Spohn }}{ }^{1,2}$, \\ Tanja Sprave 1,2, Constantinos Zamboglou ${ }^{1,2}$, Eleni Gkika ${ }^{1,2}$, Andreas Knopf ${ }^{3}$, Anca-Ligia Grosu ${ }^{1,2}$ and \\ Nils H. Nicolay $1,2, * \mathbb{D}$
}

Citation: Rühle, A.; Haehl, E.; Kalckreuth, T.; Stoian, R.; Spohn, S.K.B.; Sprave, T.; Zamboglou, C.; Gkika, E.; Knopf, A.; Grosu, A.-L.; et al. Surviving Elderly Patients with Head-and-Neck Squamous Cell Carcinoma-What Is the Long-Term Quality of Life after Curative Radiotherapy? Cancers 2021, 13, 1275. https://doi.org/10.3390/cancers 13061275

Academic Editor: Dirk Rades

Received: 17 February 2021

Accepted: 10 March 2021

Published: 13 March 2021

Publisher's Note: MDPI stays neutral with regard to jurisdictional claims in published maps and institutional affiliations.

Copyright: (c) 2021 by the authors. Licensee MDPI, Basel, Switzerland. This article is an open access article distributed under the terms and conditions of the Creative Commons Attribution (CC BY) license (https:/ / creativecommons.org/licenses/by/ $4.0 /)$.
1 Department of Radiation Oncology, University of Freiburg-University Medical Center Freiburg, Robert-Koch-Str. 3, 79106 Freiburg, Germany; alexander.ruehle@uniklinik-freiburg.de (A.R.); erik.haehl@uniklinik-freiburg.de (E.H.); tobias.kalckreuth@uniklinik-freiburg.de (T.K.); raluca.stoian@uniklinik-freiburg.de (R.S.); simon.spohn@uniklinik-freiburg.de (S.K.B.S.); tanja.sprave@uniklinik-freiburg.de (T.S.); constantinos.zamboglou@uniklinik-freiburg.de (C.Z.); eleni.gkika@uniklinik-freiburg.de (E.G.); anca.grosu@uniklinik-freiburg.de (A.-L.G.)

2 German Cancer Consortium (DKTK) Partner Site Freiburg, German Cancer Research Center (dkfz), Neuenheimer Feld 280, 69120 Heidelberg, Germany

3 Department of Otorhinolaryngology, University of Freiburg-University Medical Center Freiburg, Killianstr. 5, 79106 Freiburg, Germany; andreas.knopf@uniklinik-freiburg.de

* Correspondence: nils.nicolay@uniklinik-freiburg.de; Tel.: +49-761-270-94010

Simple Summary: As the long-term effects of radiotherapy on the quality of life (QoL) of elderly ( $\geq 65$ years) patients with head-and-neck squamous cell carcinoma (HNSCC) are not well understood, we examined the QoL of surviving elderly HNSCC patients who were treated with radiotherapy. In this cross-sectional study, long-term quality of life (QoL) at $\geq 1$ year after radiotherapy completion was comparable to the general German population of the same age and gender. Patients whose HNSCC was induced by human papillomavirus (HPV) exhibited a superior long-term global QoL than elderly patients with HPV-negative tumors. Interestingly, concomitant chemotherapy administration did not deteriorate patients' global QoL in the long-term. We did not observe differences in patients' health-related QoL in dependence of the treatment (definitive versus adjuvant (chemo)radiotherapy). Our data are important for clinicians treating elderly HNSCC patients as well as for elderly HNSCC patients themselves.

Abstract: The effects of radiotherapy on the long-term quality of life (QoL) of surviving elderly HNSCC patients are not well understood, therefore, we analyzed QoL in this population. A crosssectional analysis was performed at a tertiary cancer center to assess long-term QoL in elderly HNSCC patients. Eligible patients were $\geq 65$ years at the time of treatment who had to be alive for $\geq 1$ year after radiotherapy and without current anti-cancer treatment. QoL and patient satisfaction were assessed using the EORTC QLQ-C30, QLQ-H\&N35 and ZUF-8 questionnaires, respectively, and treatment-related toxicities were graded according to CTCAE (Common Terminology Criteria of Adverse Effects) v.5.0. Seventy-four patients met the inclusion criteria, of which 50 consented to participate. Median time between radiotherapy and QoL assessment was 32 months (range 12-113). The QLQ-C30 global QoL median amounted to 66.7 points (interquartile range (IQR) 50.0-83.3), which was comparable to the age- and gender-adjusted German population (median 65.3). Median global QoL was similar between patients undergoing definitive (75.0, IQR 50.0-83.3) and adjuvant (chemo)radiotherapy (66.7, IQR 41.7-83.3, $p=0.219)$. HPV-positive HNSCC patients had superior global QoL after radiotherapy than their HPV-negative counterparts $(p<0.05)$, and concomitant chemotherapy did not influence the long-term QoL $(p=0.966)$. Median global QoL did not correspond with physician-assessed highest-graded chronic toxicities $(p=0.640)$. The ZUF- 8 ranged at 29 points in median (IQR 27-31), showing high patient satisfaction. Surviving elderly HNSCC patients treated by radiotherapy exhibit a relatively high long-term global QoL which is a relevant information for clinicians treating elderly HNSCC patients. 
Keywords: head-and-neck cancer; head-and-neck squamous cell carcinoma; radiotherapy; chemotherapy; quality of life; patient-reported outcomes; elderly patients; geriatric patients

\section{Introduction}

Head-and-neck squamous cell carcinoma (HNSCC) constitutes a frequent malignancy and causes more than 300,000 deaths per year globally [1]. The average age of HNSCC patients at the time of diagnosis ranges between 60 and 70 years, and the percentage of elderly HNSCC patients is believed to significantly increase over the next decades [2-5]. Both clinical and tumor-associated characteristics differ between younger and elderly HNSCC patients; for instance, the female-male-distribution is shifted towards female patients and the prevalence of HPV-related carcinomas is lower in elderly HNSCC patients [5]. In addition, the benefit of both concomitant chemotherapy and epidermal growth factor receptor antibody treatment is continuously decreasing with higher age [6,7]. As elderly HNSCC patients rate their quality of life (QoL) and cure rates differently than their younger counterparts, detailed information about long-term QoL after curative treatment is needed for this cohort to enable shared decision-making based on comprehensive prognostic and QoL data for aggressive cancer treatments of HNSCC patients [8-11]. Elderly HNSCCs patients weight treatment-related toxicities and use coping strategies in a different manner compared to younger patients [12], complicating the translation of QoL studies that included all patients irrespectively of their age. In addition, physicians often inapplicably extrapolate from treatment-related toxicities on patients' general QoL, wherefore patient-reported QoL information are required [13,14].

The majority of studies which examined QoL of surviving elderly HNSCC patients focused on patients who underwent surgery, and the proportion of patients undergoing definitive (chemo)radiotherapy was relatively small [15-19]. There are only few studies that had a special focus on elderly HNSCC patients receiving (chemo)radiotherapy, and some of these studies incorporated patients with out-of-date radiotherapy techniques such as 2D or 3D radiotherapy treatments [20,21]. Furthermore, many studies focused on peritherapeutic QoL alterations, and the information about the long-term effects of radiotherapy treatment on patients' QoL in the elderly population are limited [22].

We therefore aimed to examine the long-term QoL of surviving elderly HNSCC patients after curative definitive or adjuvant (chemo)radiotherapy in a large tertiary cancer center.

\section{Results}

\subsection{Long-Term QoL Is Comparable between Surviving Elderly HNSCC Patients and the General Population}

The EORTC QLQ-C30 containing 5 functional scales (physical, role, cognitive, emotional and social), 3 symptom scales (fatigue, pain and nausea/vomiting), a global QoL scale and 6 single items (dyspnea, insomnia, appetite loss, constipation, diarrhea and financial difficulties) was used to assess patient-reported QoL in surviving elderly HNSCC patients after (chemo)radiotherapy. In order to compare the global QoL as well as the functional and symptom EORTC QLQ-C30 subscales of our elderly HNSCC cohort with the general German population of the same age and gender, we used the recently published data of the EORTC QoL Group incorporating the data of about 1000 German people [23]. The median/mean global QoL of our elderly HNSCC patient cohort amounted to 66.7/63.4 points, while the median/mean global QoL of the matched German population was 65.3/64.6 points (Figure 1A). The largest difference between our cohort consisting of elderly surviving HNSCC patients and the German reference population was observed regarding the domain of social functioning: Here, the mean value was 14.2 points higher in the age- and gender-adjusted German population (85.2 versus 71.0), which is considered a clinically meaningful difference and indicates an impaired social functioning of surviving 
elderly HNSCC patients after (chemo)radiotherapy. The remaining functional subscales did not show clinically meaningful differences between elderly HNSCC patients and the age-adjusted general population. Additionally, we compared the individual symptom subscales/items of the EORTC QLQ-C30 between both cohorts (Figure 1B): Here, appetite loss (19.0 versus 7.8$)$ and constipation (19.7 v.s. 7.1$)$ were more apparent in surviving elderly HNSCC patients compared to the general population. Interestingly, pain was in turn less pronounced in elderly HNSCC patients (22.0 v.s. 32.6).

A

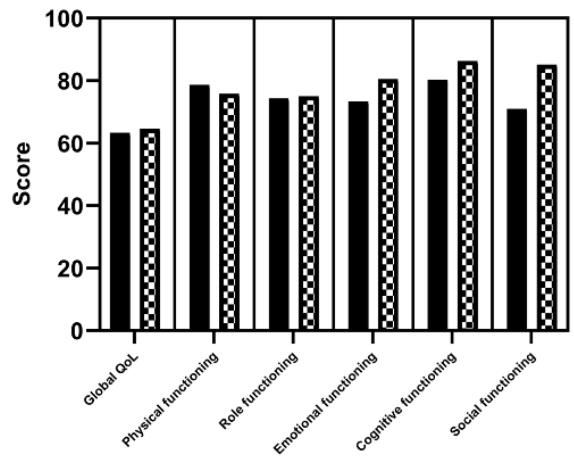

Surviving elderly HNSCC patients

W German age- and gender-matched population

B

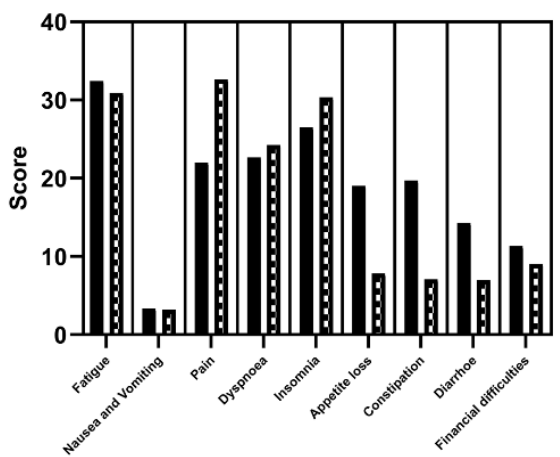

Surviving elderly HNSCC patients

Werman age- and gender-matched population

Figure 1. Comparison between surviving elderly HNSCC (head-and-neck squamous cell carcinoma) patients and matched patients from the German general population (with the same age and gender) regarding the functioning subscales (A) and the symptom subscales/items (B) of the EORTC QLQ-C30 questionnaire. Bars represent the mean values of the different subscales. General population norm data for Germany are obtained from [23].

\subsection{HPV Positivity and Performance Status Correspond with Long-Term QoL in Surviving Elderly HNSCC Patients}

We examined potential influences of patients' gender, smoking status and HPV positivity on the long-term QoL of surviving elderly HNSCC patients. There was a trend towards decreased global QoL in female patients ( $p=0.097$, Mann-Whitney U test) (Figure 2A), which can also be observed within the general population. While the median global QoL was comparable between smokers and non-smokers $(p=0.248$, Mann-Whitney U test) (Figure 2B), elderly patients with HPV-positive tumors exhibited considerably increased global QoL values status ( $p<0.05$, Mann-Whitney U test) (Figure 2C). The median/mean global QoL was 50.0/51.1 points in HPV-negative HNSCC patients, while it amounted to 83.3/72.2 points in HPV-positive patients. The baseline performance status prior to the initiation of radiotherapy was observed to moderately correlate with the global QoL of the surviving patients $(\varrho=0.390, p<0.01$ ) (Figure 2D), whereas such an association could not be demonstrated for the patients' comorbidity burden as measured by the age-adjusted Charlson Comorbidity Index ( $\varrho=-0.198, p=0.177$ ) (Figure 2E). 
A

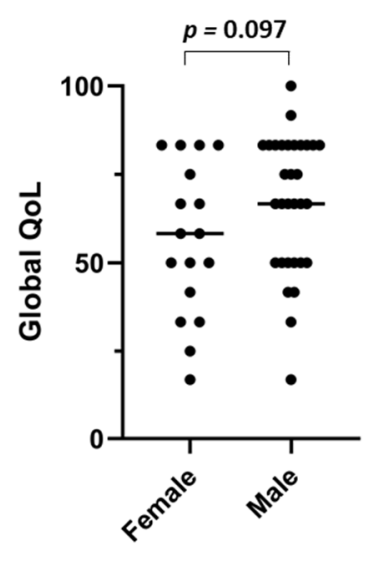

B

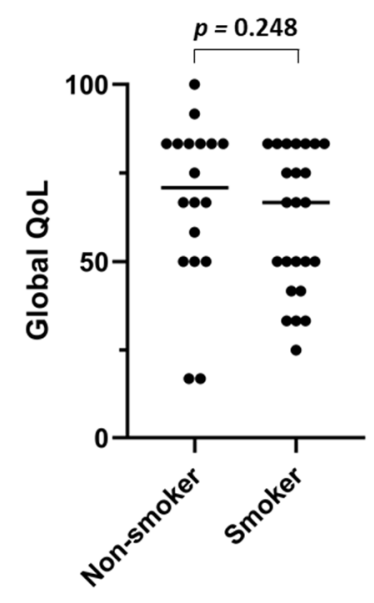

C

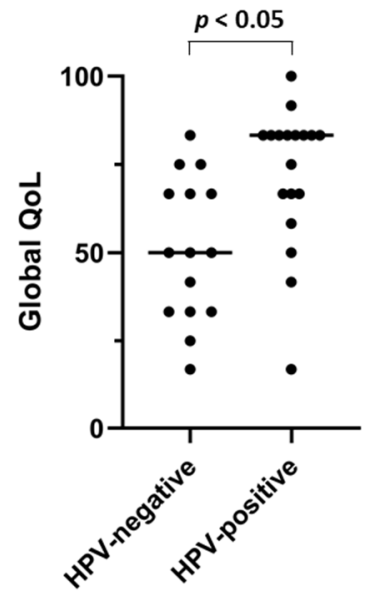

D

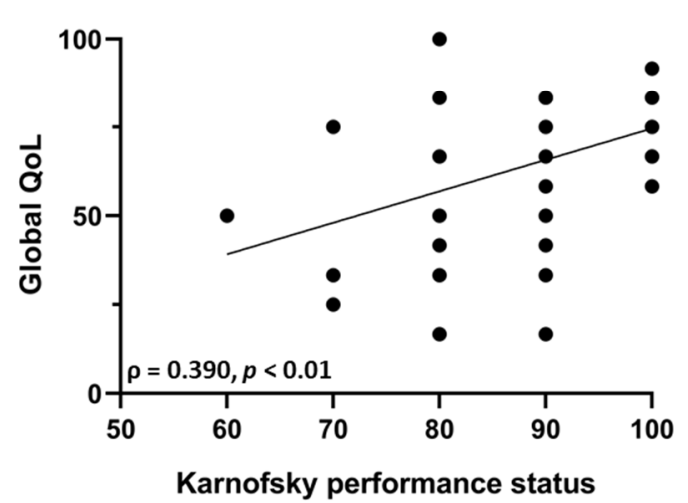

E

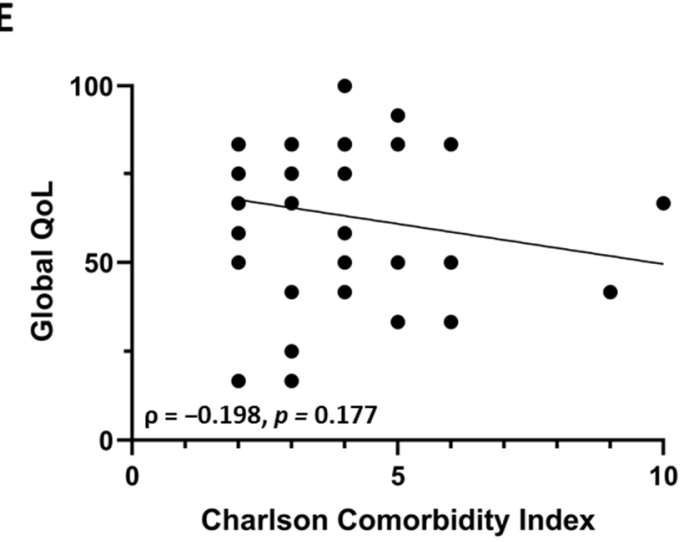

Figure 2. Global QoL of surviving elderly HNSCC patients undergoing (chemo)radiotherapy in terms of patients' gender (A), smoking status (B) and HPV status (C). Correlation between global QoL and Karnofsky performance status (D) or Charlson Comorbidity Index (E). (A-C) Each scatter plot represents one patient, and the line shows the median value. Groups were compared using Mann-Whitney U tests. (D,E) Spearman's rho with the corresponding $p$-value is indicated for the correlation analyses. The line shows the regression line of the xy-matrix.

2.3. Prior Tumor Resection and Concomitant Chemotherapy Do Not Influence Long-Term QoL in Surviving Elderly HNSCC Patients

Based on the EORTC QLQ-C30 and EORTC QLQ-H\&N35 questionnaires, potential influences of tumor surgery prior to radiotherapy or the addition of concomitant chemotherapy on the long-term QoL were assessed. The majority of EORTC QLQ-H\&N35 symptom subscales showed comparable score values between the definitive and adjuvant radiotherapy group; however, patients reported less symptoms regarding "troubles with social eating" $(p<0.05)$ and mouth opening $(p<0.01)$ without prior tumor resection (Figure $3 \mathrm{~A}$ ). In contrast, patients who received primary resection with adjuvant (chemo)radiotherapy had fewer problems with reduced sexuality than patients undergoing primary (chemo)radiotherapy $(p<0.01)$. Neither global long-term QoL nor patient satisfaction, as measured by the ZUF-8 questionnaire, differed significantly between both groups ( $p=0.219$ for both categories) (Figure 3B,C). The long-term swallowing function was the only reported symptom that significantly differed between the radio- and chemoradiotherapy group ( $p<0.05$, in favor of radiotherapy) (Figure 3D). In addition, concomitant chemotherapy administration had no long-term effect on the QoL $(p=0.966)$ and patient satisfaction $(p=0.709)$ of surviving elderly HNSCC patients (Figure 3E,F). 
A

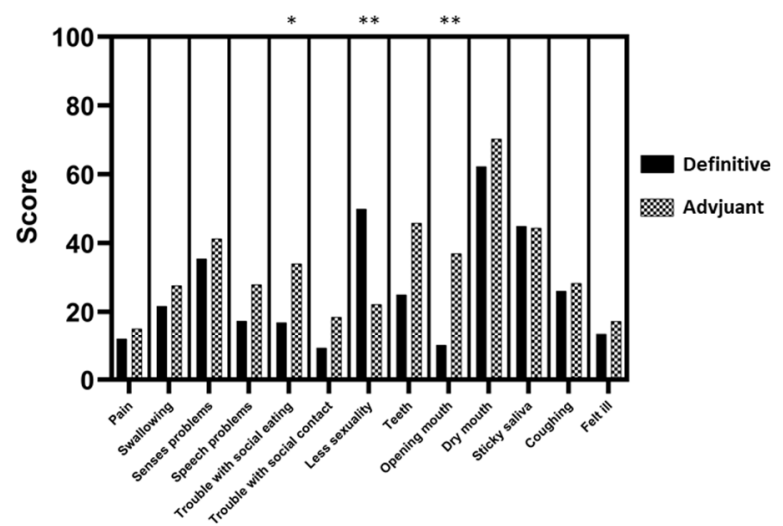

D

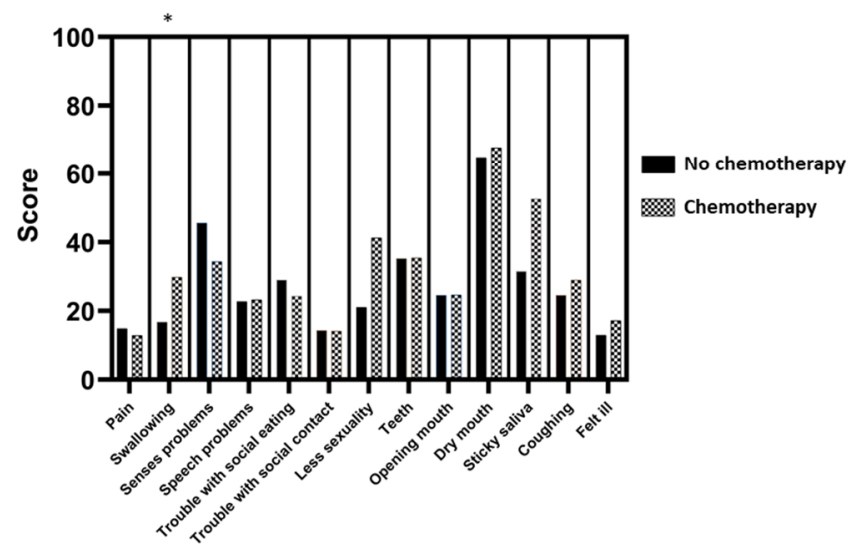

B

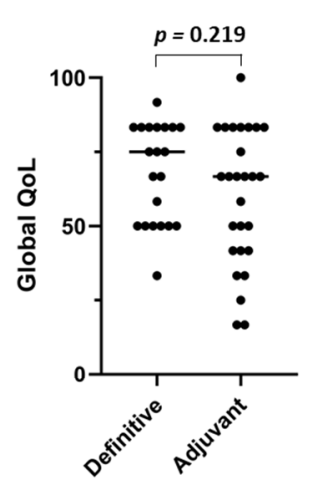

E

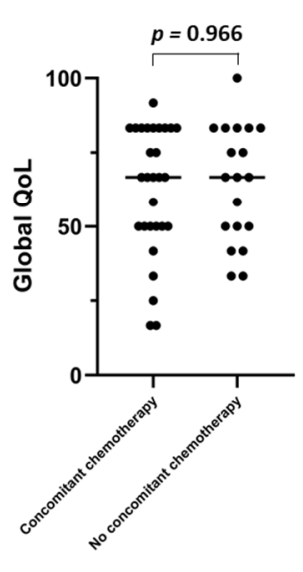

C

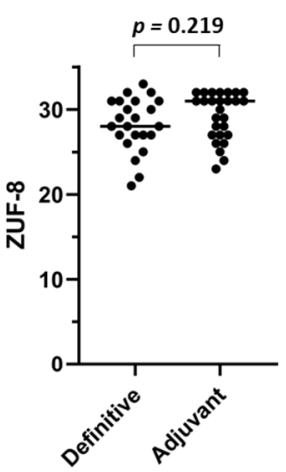

$\mathbf{F}$

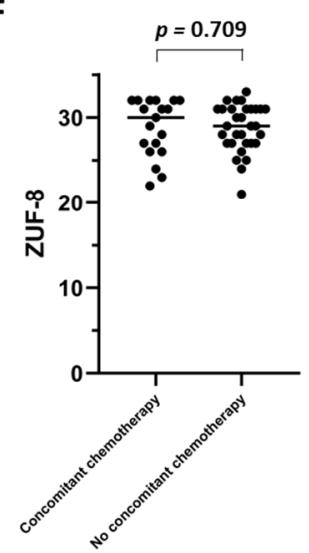

Figure 3. Comparison between the definitive and adjuvant (chemo)radiotherapy group regarding the different EORTC QLQ-H\&N35 symptom subscales (A), general QoL (B) and patient satisfaction measured by the ZUF-8 questionnaire (C) of surviving elderly HNSCC patients. Influence of concomitant chemotherapy administration in surviving elderly HNSCC patients in terms of the EORTC QLQ-H\&N35 symptom subscales (D), general QoL (E) and patient satisfaction (F). Groups were compared using Mann-Whitney U tests. ${ }^{*} p<0.05,{ }^{* *} p<0.01$.

\subsection{Long-Term QoL in Surviving Elderly HNSCC Patients Does Not Correlate with Physician-Assessed CTCAE-Based Chronic Toxicities}

The vast majority of patients exhibited either no $(n=2,4 \%)$ or only CTCAE (Common Terminology Criteria of Adverse Effects) grade 1/2 ( $n=41,82 \%)$ chronic toxicities (Table 1). A total of 7 patients (14\%) suffered from severe (CTCAE grade 3 ) toxicities; however, there was no significant difference in terms of the long-term global QoL in dependence of the maximum physician-assessed chronic toxicity ( $p=0.640$, Kruskal-Wallis test). While the median global QoL amounted to 70.9 and 66.7 points in patients with grade 1 and grade 2 toxicities, respectively, it was 66.7 points in patients with grade 3 chronic toxicities. Similarly, patient satisfaction did not differ depending on patients' chronic toxicities ( $p=0.517$, Kruskal-Wallis test).

Table 1. Median global QoL and patient satisfaction in dependence of the highest-graded chronic toxicity according to CTCAE (Common Terminology Criteria of Adverse Effects) version 5.0. $p$-values are derived from Kruskal-Wallis tests.

\begin{tabular}{ccccc}
\hline & Global QoL (QLQ-C30) & $p$-Value & ZUF-8 & $p$-Value \\
\hline $0(n=2)$ & 66.7 & & 26.5 & \\
$1(n=14)$ & 70.9 & & 30.5 & \\
$2(n=27)$ & 66.7 & & 29.0 & \\
$3(n=7)$ & 66.7 & 0.640 & 29.0 & 0.517 \\
\hline
\end{tabular}


The majority of surviving elderly HNSCC did not use analgesic medication $(n=40$, $80 \%$ ) or nutritional supplements ( $n=40,80 \%$ ) within the last week prior to questionnaire completion, and this rate was comparable between the definitive and adjuvant (chemo)radiotherapy groups (Table 2). While none of the 23 patients in the definitive treatment cohort had a long-term feeding tube dependence, 4 of 27 patients in the adjuvant treatment group required a permanent feeding tube ( $p=0.115$, Fisher's exact test).

Table 2. Comparison between the definitive and adjuvant (chemo)radiotherapy group in terms of pain killer usage, nutritional supplement intake and feeding tube dependence (within the last week) among surviving elderly HNSCC patients. $p$-values are obtained from Fisher's exact tests.

\begin{tabular}{cccc}
\hline & $\begin{array}{c}\text { Definitive } \\
\text { (chemo)radiotherapy }\end{array}$ & $\begin{array}{c}\text { Adjuvant } \\
\text { (chemo)radiotherapy }\end{array}$ & $p$-Value \\
\hline No pain killer usage & 19 & 21 & \\
Pain killer usage & 4 & 6 & 0.736 \\
\hline No nutritional supplement intake & 19 & 21 & 0.736 \\
Nutritional supplement intake & 4 & 6 & \\
\hline No feeding tube dependence & 23 & 23 & 0.115 \\
Feeding tube dependence & 0 & 4 & \\
\hline
\end{tabular}

\section{Discussion}

In this cross-sectional analysis including 50 surviving elderly HNSCC patients who received (chemo)radiotherapy at a large tertiary cancer center, we could demonstrate a global QoL that was comparable to the age- and gender-adjusted German general population. Furthermore, we did not observe statistically different global QoL values between definitive and adjuvant (chemo)radiotherapy or between radiotherapy and concomitant chemoradiotherapy. Surviving elderly HPV-positive patients were found to exhibit a superior QoL than their HPV-negative counterparts, which is an important fact regarding current de-escalation debates for HPV-positive patients. Overall, our findings may support clinicians in counselling elderly HNSCC patients for curative therapies regarding their long-term QoL.

Previous studies assessing patient-reported outcomes after HNSCC treatment could show equivalent health-related QoL compared to the age- and gender-matched population in younger patients, but to date, no information has been available for the distinct subgroup of elderly patients, for whom QoL is deemed considerably more important [24,25]. To the best of our knowledge, we present, for the first time, evidence that this observation of equivalent QoL values holds also true for the elderly HNSCC population treated by (chemo)radiotherapy. The subscale value for pain was even lower by more than 10 points in elderly HNSCC survivors than in the age- and gender-controlled general German population. While fatigue is a frequent long-term sequela for several cancer entities such as stomach, lung and breast cancer [26,27], it seems to be a less common patient-reported adverse event in surviving elderly HNSCC patients after (chemo)radiotherapy when comparing with the general population. In this context, it should be noted that most HNSCC patients adapt to their physical impairments such as xerostomia or dysphagia, leading to sometimes better global QoL values than physicians would assume. For instance, longterm QoL is not reduced after laryngectomy, contrary to common physician belief [28]. Although the global QoL as well as the physical, role, emotional and cognitive functioning were comparable between elderly HNSCC patients and the age- and gender-matched population, there was a considerable decrease of surviving HNSCC patients' social functioning. Previous studies have also reported that social functioning was the worse functioning scale of surviving HNSCC patients after radiotherapy [20,21].

In a prospective study of Derks and coworkers, elderly HNSCC patients ( $\geq 70$ years) exhibited comparable global QoL values to patients aged between 45 and 60 years at one year after treatment [17]. In contrast, Laraway and colleagues could demonstrate for 
surgical HNSCC treatments that several QoL domains such as physical and emotional function were superior in HNSCC patients aged 65 years and older compared to younger patients [19]. One hypothesis for these observations may be that elderly patients have less lifetime to lose and that their expectations regarding outcome are lower compared to their younger counterparts.

Interestingly, elderly HPV-positive HNSCC patients were found to have a considerably higher QoL than HPV-negative patients in our study. There are several possible explanations for this observation: In general, HPV-positive patients exhibit a higher socioeconomic status, increased financial income and higher education level which may all contribute to a usually superior QoL in this cohort [29]. In a substudy of the Trans-Tasman Radiation Oncology Group (TROG) 02.02 trial, HPV-positive oropharyngeal carcinoma patients were demonstrated to have elevated baseline QoL values but a more pronounced decline during chemoradiotherapy; however, they again had a better QoL than HPV-negative oropharyngeal cancer patients at 12 months after treatment [30]. This finding was confirmed in other analyses for long-term surviving patients with HPV-positive HNSCC [31,32]. The high global QoL results of surviving elderly HNSCC patients after (chemo)radiotherapy should be considered in the current treatment de-escalation debates.

In our analysis, simultaneous chemoradiotherapy resulted in similar global QoL, headand-neck cancer-related symptom severity and patient satisfaction compared to patients receiving radiotherapy alone. Chemotherapy administered either in the definitive setting or postoperatively due to positive resection margins or extranodal extension remains controversial, especially in the postoperative setting. Neither the EORTC 22931 nor the RTOG 9501 trials that established the criteria for postoperative chemoradiotherapy did provide sufficient evidence for the usage of concomitant chemotherapy in the elderly, either due to complete exclusion of patients above 70 years (EORTC 22931) or due to low numbers $(n=25)$ of patients aged 70 years or older (RTOG 9501) [33,34]. The previous retrospective National Cancer Data Base (NCDB) and the Medicare-linked Surveillance, Epidemiology, and End Results (SEER) database analyses could not demonstrate a benefit for adding chemotherapy to postoperative radiotherapy in the case of positive resection margins or extranodal extension [35-37]. Our results revealed at least no signs of significantly deteriorated long-term QoL in surviving elderly HNSCC patients after chemoradiotherapy; only patient-reported swallowing dysfunction was found to be significantly higher (and clinically significant with a difference $>10$ points) in the chemoradiotherapy group. This finding is in line with a previous study in which patients undergoing chemoradiotherapy exhibited swallowing disorders more frequently than patients treated by radiotherapy alone [38].

In our analysis, we could not detect a significant difference regarding patients' longterm global QoL in dependence of the treatment modality. However, social eating as well as mouth opening showed clinically meaningful and significantly preferable values for the definitive (chemo)radiotherapy group which is in line with findings of other studies that reported superior QoL, in particular swallowing, social eating, speech and taste of patients undergoing definitive (chemo)radiotherapy compared to patients treated by surgery plus adjuvant (chemo)radiotherapy [39,40]. It should be also emphasized that results of previous studies concerning the long-term QoL of HNSCC patients after radiotherapy are difficult to transfer to the current situation, in which high-precision intensity-modulated and imageguided radiotherapy define the state-of-the art treatment. For instance, Huang et al. could prove the superiority of these modern techniques in regards to a better swallowing-related QoL of surviving HNSCC patients [20].

No association between physician-assessed chronic toxicities according to CTCAE and global QoL in our cohort of surviving elderly HNSCC patients could be observed. There were patients with only mild or moderate chronic toxicities (CTCAE grade 1-2) and a considerably reduced long-term global QoL, and vice versa, patients with severe chronic toxicities (CTCAE grade 3) were found to exhibit very high QoL scores. The DAHANCA group compared physician-reported and patient-reported outcome measures 
and observed a significant correlation of some observer-based toxicities such as dysphagia with patient-reported global QoL, whereas other chronic toxicities such as fibrosis and oedema did not correlate with patients' global health status [41]. It has been reported that physicians commonly focus on higher-grade (CTCAE grade $\geq 3$ ) toxicities and underestimate the cumulative impact of lower-grade toxicities on the patient-reported health status [42]. Following the promising feasibility and clinical results from prospective trials that investigated patient-reported outcomes, many institutions are currently integrating patient-reported outcome measures into the clinical routine, and previous studies also demonstrated the feasibility to perform app-based electronic patient-reported outcome measures in HNSCC patients [43-46].

Despite providing important information for clinicians regarding the long-term QoL of surviving elderly HNSCC patients after radiotherapy, our analysis has some limitations. Due to the cross-sectional study design, we were not able to longitudinally assess patients QoL and compare to baseline values prior to radiotherapy. In addition, patients who agreed to participate in the study may exhibit differences in their QoL compared to patients who declined participation $(n=7)$ or failed to return the questionnaire $(n=4)$. We furthermore only focused our analysis on patients who were free from active cancer and did not receive any antineoplastic treatment, so the generalizability to patients with persistent or recurrent disease is limited. Mild-to-moderate toxicity rates are known to be underreported in retrospective assessments compared to prospective trials, which is a caveat of our study; in addition, the exact onset of these toxicities could not be accurately assessed due to the crosssectional character of this analysis. At last, it should be acknowledged that extrapolation of our results to the situation in other countries is not obvious, as patient-reported QoL data are subject to considerable intercultural and interethnic differences [47,48], pointing out the necessity for multinational studies in the future.

\section{Materials and Methods}

\subsection{Patients and Treatment}

The Independent Ethics Committee of the University of Freiburg approved this crosssectional study in advance (reference no. 371/20), and written informed consent was obtained from all patients who agreed to participate.

HNSCC patients aged $\geq 65$ years who were treated with curative (chemo)radiotherapy between 2010 and 2019 at the Department of Radiation Oncology, University of Freiburg Medical Center, and who were still alive at one year after radiotherapy and not lost to follow-up, were eligible for this study. Seventy-four patients met the inclusion criteria and we attempted to inform them about the QoL study. Fifty-four patients agreed to participate, of which 50 answered the required questionnaires. The CONSORT diagram for the patient screening is demonstrated in Figure 4.

Demographic and treatment characteristics of these patients were obtained from the electronic patient records. Patients with a smoking history of at least 10 pack/years were considered as smokers. Treatment decisions for all HNSCC patients were based on multidisciplinary tumor board recommendations. Patients who received definitive (chemo)radiotherapy received doses ranging at $70 \mathrm{~Gy}_{\mathrm{EQD} 2}$ to the primary tumor, whereas patients who received adjuvant (chemo)radiotherapy received 60-66 GyеQD2 to the tumor bed. Photon radiotherapy was carried out using intensity-modulated radiotherapy, volumetric modulated arc therapy or tomotherapy (Figure 5). 


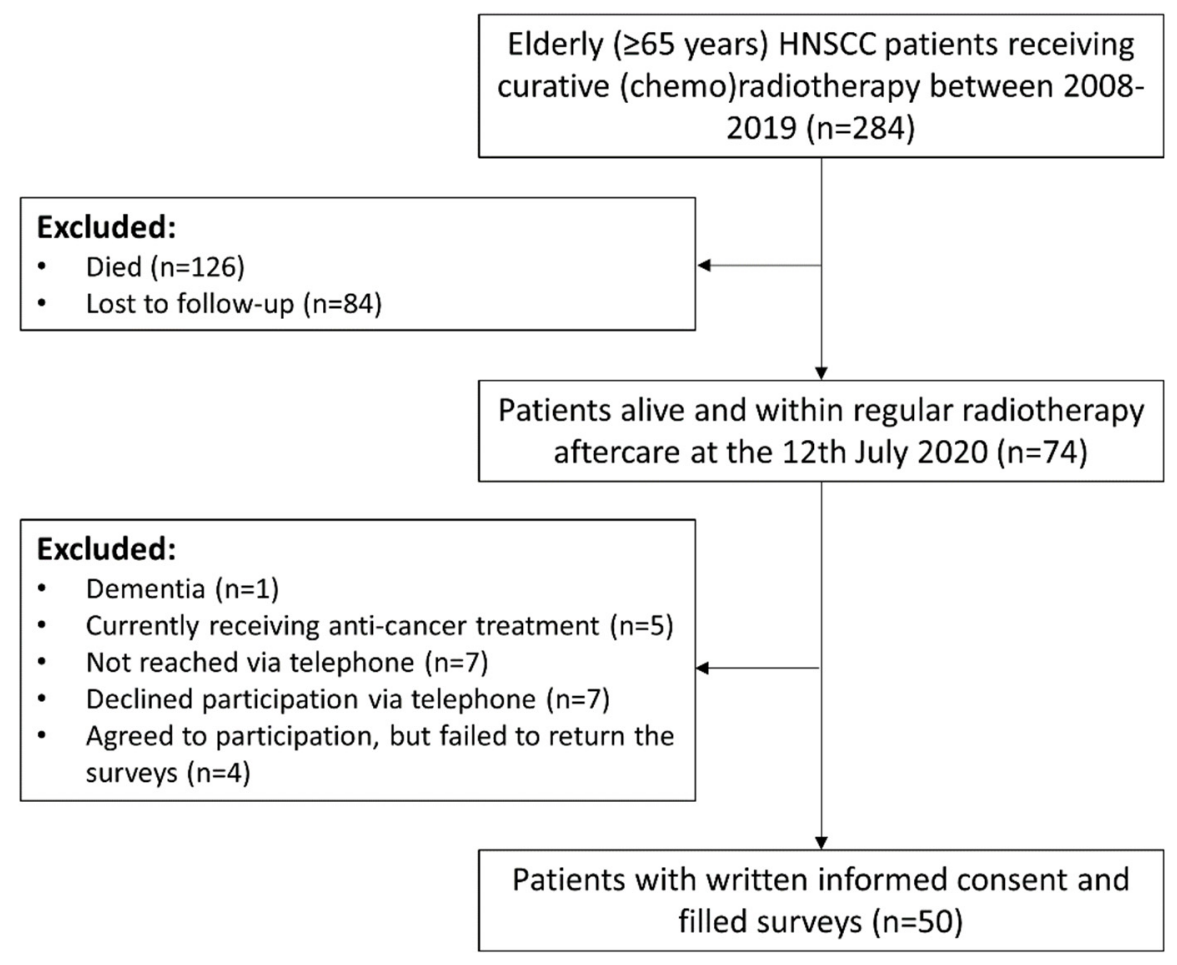

Figure 4. CONSORT flow diagram showing the enrollment of participants in the study.

In case of no medical contraindications, definitive radiotherapy was accompanied by concurrent chemotherapy for all patients with locally advanced HNSCC in the definitive treatment situation. Patients who were treated with primary surgery received concomitant chemoradiotherapy in the event of incomplete resection or extranodal extension.

\subsection{Toxicity Analyses}

Chronic treatment-related toxicities were classified in accordance with the Common Terminology Criteria of Adverse Effects (CTCAE) version 5.0. All toxicities occurring later than 90 days after radiotherapy were considered as chronic toxicities. Feeding tube dependence, usage of pain killers and intake of nutrition supplements were extracted from the EORTC H\&N35 module.

\subsection{Patient and Tumor Characteristics}

A total of 50 patients agreed to participate in this study and filled out paper-based QoL and patient satisfaction questionnaires. The median age of the cohort at the time of treatment initiation was 69 years (range 65-82 years), and the majority of patients were male $(n=32,64 \%)$. Most patients had a Karnofsky Performance Status between $90 \%$ and $100 \%(n=33,66 \%)$ at the time of radiotherapy, and the comorbidity burden, quantified by the age-adjusted CCI, was modest and shown by a median CCI value of 3 (range 2-10). Tumors were most commonly located in the oropharynx $(n=26,52 \%)$, followed by the oral cavity $(n=9,18 \%)$ and the larynx $(n=6,12 \%)$. Twenty-three patients $(46 \%)$ underwent definitive (chemo)radiotherapy (20 with definitive chemoradiotherapy and 3 with definitive radiotherapy), while 27 patients $(54 \%$ ) received (chemo)radiotherapy after primary surgery. Of these 27 patients, 16 received adjuvant radiotherapy, while 11 underwent adjuvant/additive chemoradiotherapy either due to R1 resection $(n=1)$ or due to extranodal extension ( $n=11$; one patient exhibited both R1 resection and extranodal extension). As described previously, completion rates for both radiotherapy and chemotherapy were very high with $96 \%$ and $97 \%$, respectively $[49,50]$. Detailed patient and treatment characteristics can be found in Table 3 . 

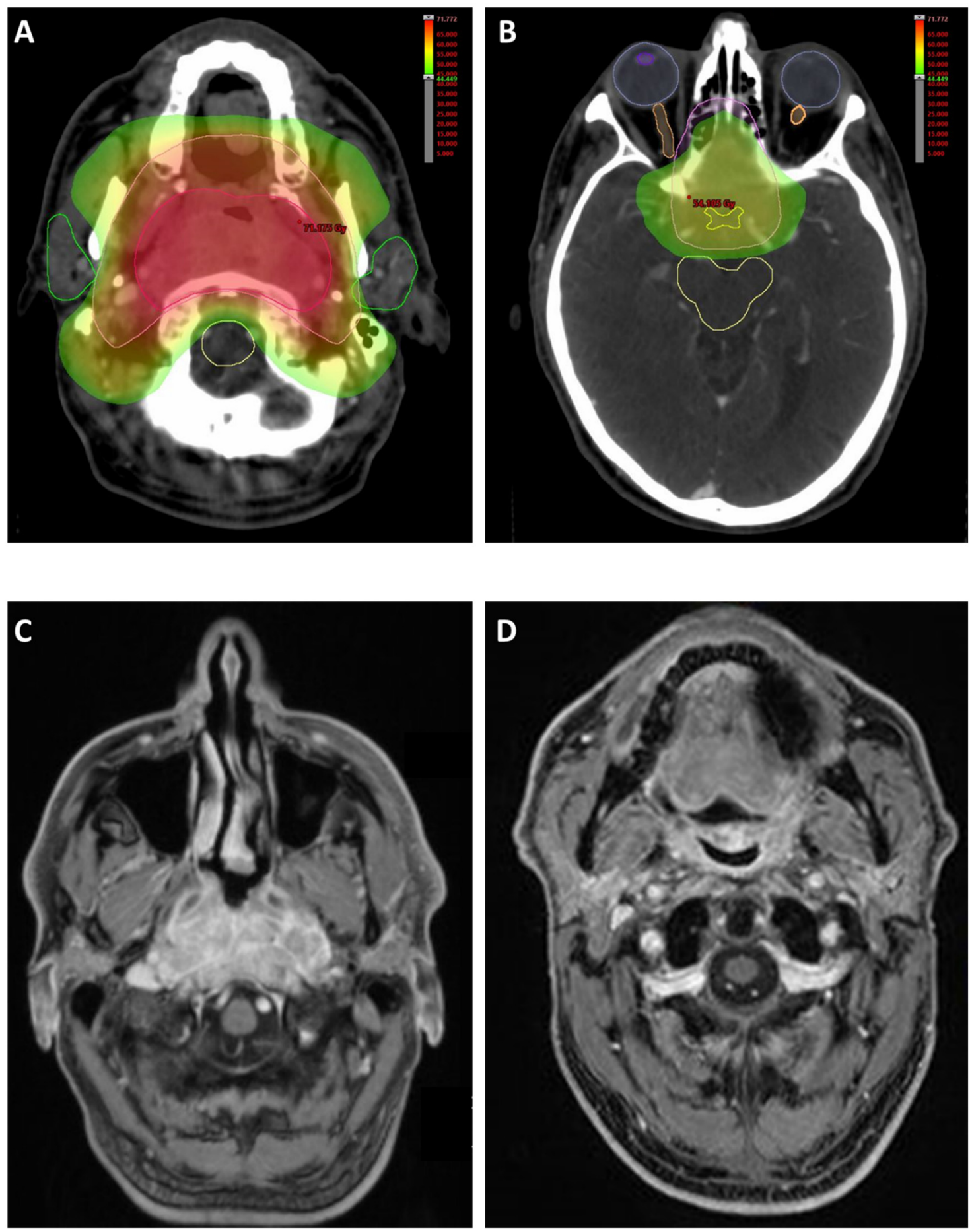

Figure 5. 67-year-old male patient with an EBV-positive nasopharyngeal squamous cell carcinoma, infiltrating the sella turcica, clivus and right-sided internal carotid artery and affecting the chiasma, cT4 cN0 cM0, G3. Definitive cisplatinbased chemoradiotherapy was applied between May and July 2018. The primary tumor received 69.4 Gy in 37 fractions (14 fractions with 2 Gy and 23 fractions with 1.8 Gy). (A,B) Axial images showing bilateral parotid gland and spinal cord protection. (C) Contrast-enhanced MRI showing the nasopharyngeal carcinoma prior to radiotherapy. (D) Follow-up image at 2.5 years later (September 2020) showing no signs of recurrence. 
Table 3. Patient and treatment characteristics of the study cohort consisting of 50 elderly HNSCC patients treated by (chemo)radiotherapy in our institution between 2010 and 2019. CCI = Charlson Comorbidity Index, HPV = Human papillomavirus.

\begin{tabular}{|c|c|c|c|}
\hline \multicolumn{2}{|c|}{ Parameter } & \multicolumn{2}{|c|}{ Median (Range) } \\
\hline \multicolumn{2}{|c|}{ Age at Radiotherapy } & \multicolumn{2}{|c|}{69 Years $(65-82)$} \\
\hline & & $n$ & $\%$ \\
\hline \multirow[t]{2}{*}{ Gender } & male & 32 & 64 \\
\hline & female & 18 & 36 \\
\hline \multirow[t]{3}{*}{ Smoking } & non-smoker & 19 & 38 \\
\hline & smoker & 24 & 48 \\
\hline & missing & 7 & 14 \\
\hline \multirow[t]{5}{*}{ Karnofsky performance status } & $100 \%$ & 8 & 16 \\
\hline & $90 \%$ & 25 & 50 \\
\hline & $80 \%$ & 13 & 26 \\
\hline & $70 \%$ & 3 & 6 \\
\hline & $60 \%$ & 1 & 2 \\
\hline \multirow[t]{7}{*}{ CCI } & 2 & 10 & 20 \\
\hline & 3 & 17 & 34 \\
\hline & 4 & 9 & 18 \\
\hline & 5 & 5 & 10 \\
\hline & 6 & 7 & 14 \\
\hline & 9 & 1 & 2 \\
\hline & 10 & 1 & 2 \\
\hline \multirow[t]{7}{*}{ Localization } & nasopharynx & 1 & 2 \\
\hline & oropharynx & 26 & 52 \\
\hline & hypopharynx & 1 & 2 \\
\hline & oral cavity & 9 & 18 \\
\hline & larynx & 6 & 12 \\
\hline & multi-level & 3 & 6 \\
\hline & others & 4 & 8 \\
\hline \multirow[t]{4}{*}{ T stage } & $\mathrm{T} 1$ & 12 & 24 \\
\hline & $\mathrm{T} 2$ & 17 & 34 \\
\hline & T3 & 12 & 24 \\
\hline & $\mathrm{T} 4$ & 9 & 18 \\
\hline \multirow[t]{4}{*}{ n stage } & No & 16 & 32 \\
\hline & N1 & 11 & 22 \\
\hline & N2 & 18 & 36 \\
\hline & N3 & 5 & 10 \\
\hline \multirow[t]{4}{*}{ Grading } & G1 & 2 & 4 \\
\hline & G2 & 23 & 46 \\
\hline & G3 & 24 & 48 \\
\hline & missing & 1 & 2 \\
\hline \multirow[t]{3}{*}{ HPV } & HPV-negative & 16 & 32 \\
\hline & HPV-positive & 18 & 36 \\
\hline & missing & 16 & 32 \\
\hline \multirow[t]{2}{*}{ Radiotherapy completion } & completed & 48 & 96 \\
\hline & discontinued & 2 & 4 \\
\hline \multirow[t]{2}{*}{ Chemotherapy completion $(n=31)$} & completed & 30 & 97 \\
\hline & discontinued & 1 & 3 \\
\hline \multirow[t]{4}{*}{ Treatment concept } & definitive radiotherapy & 3 & 6 \\
\hline & definitive chemoradiotherapy & 20 & 40 \\
\hline & adjuvant radiotherapy & 16 & 32 \\
\hline & adjuvant chemoradiotherapy & 11 & 22 \\
\hline \multirow[t]{3}{*}{ Chemotherapy $(n=31)$} & platinum agents & 29 & 94 \\
\hline & nivolumab & 2 & 6 \\
\hline & & \multicolumn{2}{|c|}{ Median (range) } \\
\hline \multicolumn{2}{|c|}{ Radiation dose (definitive treatment) in EQD2 } & \multicolumn{2}{|c|}{70 Gy (55-72) } \\
\hline Radiation dose (adjuvar & treatment) in EQD2 & & \\
\hline
\end{tabular}




\subsection{QoL and Patient Satisfaction Questionnaires}

Long-term QoL was assessed with the EORTC QLQ-C30 questionnaire (version 3) and the EORTC H\&N35 module, while patient satisfaction was quantified using the ZUF-8 survey. The EORTC questionnaires were analyzed according to the EORTC scoring manual, while the ZUF-8 questionnaire as instrument for patient satisfaction with the treatment was examined as described by Schmidt et al. [51]. The ZUF-8 is the German adaption of the American CSQ-Questionnaire and consists of 8 questions with a 4-point Likert scale each, so that a score between 8 (minimal patient satisfaction) and 32 points (maximum patient satisfaction) can be achieved.

\subsection{Statistical Analysis}

Fisher's exact tests were carried out to test for potential differences within contingency tables, and either Mann-Whitney U tests (2 groups) or Kruskal-Wallis tests ( $\geq 3$ groups) were performed to compare QoL outcomes. Correlative analyses were conducted using Spearman's rank correlations. Statistical significance was assumed for $p<0.05$ throughout the study. IBM SPSS Statistics software version 25 (IBM, Armonk, NY, USA) was used for statistical analyses, and results were visualized using GraphPad Prism software version 8 (GraphPad Software, San Diego, CA, USA).

\section{Conclusions}

In this cross-sectional study involving 50 surviving elderly HNSCC patients who underwent radiotherapy at a large tertiary cancer center, we could demonstrate that patients' self-reported long-term QoL is comparable to the age- and gender-adjusted general population. Neither the choice of primary curative treatment nor addition of concomitant chemotherapy did significantly deteriorate patients' long-term global QoL or patient satisfaction. Interestingly, we could identify initial Karnofsky performance status and HPV status as parameters that were associated with post-treatment global QoL. The global QoL of surviving elderly HPV-positive HNSCC patients after radiotherapy was excellent (median global QoL = 83.3), which should be considered when discussing age-dependent treatment de-escalation for elderly HPV-positive HNSCC patients.

Author Contributions: Conceptualization, A.R. and N.H.N.; methodology, A.R. and N.H.N.; software, A.R.; data curation, A.R., E.H., T.K. and R.S.; writing—original draft preparation, A.R.; writingreview and editing, S.K.B.S., T.S., C.Z., E.G., A.K., A.-L.G. and N.H.N.; visualization, A.R. and N.H.N.; supervision, N.H.N.; project administration, N.H.N. All authors have read and agreed to the published version of the manuscript.

Funding: Alexander Rühle was supported by the IMM-PACT-Program for Clinician Scientists, Department of Medicine II, Medical Center-University of Freiburg and Faculty of Medicine, University of Freiburg, funded by the Deutsche Forschungsgemeinschaft (DFG, German Research Foundation) 413517907.

Institutional Review Board Statement: The study was conducted according to the guidelines of the Declaration of Helsinki, and approved by the Institutional Review Board (371/20, 25 June 2020).

Informed Consent Statement: Informed consent was obtained from all subjects involved in the study.

Data Availability Statement: The anonymized data presented in this study are available on request from the corresponding author. The data are not publicly available due to data protection principles.

Conflicts of Interest: The authors declare no conflict of interest.

\section{References}

1. Bray, F.; Ferlay, J.; Soerjomataram, I.; Siegel, R.L.; Torre, L.A.; Jemal, A. Global cancer statistics 2018: GLOBOCAN estimates of incidence and mortality worldwide for 36 cancers in 185 countries. CA Cancer J. Clin. 2018, 68, 394-424. [CrossRef] [PubMed]

2. Grenman, R.; Chevalier, D.; Gregoire, V.; Myers, E.; Rogers, S. Treatment of head and neck cancer in the elderly: European Consensus (panel 6) at the EUFOS Congress in Vienna 2007. Eur. Arch. Otorhinolaryngol. 2010, 267, 1619-1621. [CrossRef] [PubMed] 
3. Vigneswaran, N.; Williams, M.D. Epidemiologic trends in head and neck cancer and aids in diagnosis. Oral Maxillofac. Surg. Clin. N. Am. 2014, 26, 123-141. [CrossRef] [PubMed]

4. Smith, B.D.; Smith, G.L.; Hurria, A.; Hortobagyi, G.N.; Buchholz, T.A. Future of cancer incidence in the United States: Burdens upon an aging, changing nation. J. Clin. Oncol. 2009, 27, 2758-2765. [CrossRef] [PubMed]

5. Gugić, J.; Strojan, P. Squamous cell carcinoma of the head and neck in the elderly. Rep. Pract. Oncol Radiother. 2012, 18, 16-25. [CrossRef]

6. Lacas, B.; Carmel, A.; Landais, C.; Wong, S.J.; Licitra, L.; Tobias, J.S.; Burtness, B.; Grazia Ghi, M.; Cohen, E.E.W.; Grau, C.; et al. Meta-analysis of chemotherapy in head and neck cancer (MACH-NC): An update on 107 randomized trials and 19805 patients, on behalf of MACH-NC group. Radiother Oncol. 2021. [CrossRef] [PubMed]

7. Bonner, J.A.; Harari, P.M.; Giralt, J.; Cohen, R.B.; Jones, C.U.; Sur, R.K.; Raben, D.; Baselga, J.; Spencer, S.A.; Zhu, J.; et al. Radiotherapy plus cetuximab for locoregionally advanced head and neck cancer: 5 -year survival data from a phase 3 randomised trial, and relation between cetuximab-induced rash and survival. Lancet Oncol. 2010, 11, 21-28. [CrossRef]

8. Yellen, S.B.; Cella, D.F.; Leslie, W.T. Age and clinical decision making in oncology patients. J. Natl. Cancer Inst. 1994, 86, 1766-1770. [CrossRef]

9. Rühle, A.; Haehl, E.; David, H.; Kalckreuth, T.; Sprave, T.; Stoian, R.; Zamboglou, C.; Gkika, E.; Knopf, A.; Grosu, A.L.; et al. The Value of Laboratory Parameters for Anemia, Renal Function, Systemic Inflammation and Nutritional Status as Predictors for Outcome in Elderly Patients with Head-and-Neck Cancers. Cancers 2020, 12, 1698. [CrossRef]

10. Rühle, A.; Stromberger, C.; Haehl, E.; Senger, C.; David, H.; Stoian, R.; Zamboglou, C.; Knopf, A.; Budach, V.; Grosu, A.L.; et al. Development and validation of a novel prognostic score for elderly head-and-neck cancer patients undergoing radiotherapy or chemoradiation. Radiother. Oncol. 2020, 154, 276-282. [CrossRef] [PubMed]

11. Sprave, T.; Zamboglou, C.; Verma, V.; Nicolay, N.H.; Grosu, A.L.; Lindenmeier, J.; Tscheulin, D.K. Characterization of healthrelated quality of life based on the EQ-5D-5L questionnaire in head-and-neck cancer patients undergoing modern radiotherapy. Expert Rev. Pharmacoecon. Outcomes Res. 2020, 20, 673-682. [CrossRef]

12. Derks, W.; Leeuw, J.R.; Hordijk, G.J.; Winnubst, J.A. Differences in coping style and locus of control between older and younger patients with head and neck cancer. Clin. Otolaryngol. 2005, 30, 186-192. [CrossRef] [PubMed]

13. Kahn, S.B.; Houts, P.S.; Harding, S.P. Quality of life and patients with cancer: A comparative study of patient versus physician perceptions and its implications for cancer education. J. Cancer Educ. 1992, 7, 241-249. [CrossRef] [PubMed]

14. Lipscomb, J.; Gotay, C.C.; Snyder, C.F. Patient-reported outcomes in cancer: A review of recent research and policy initiatives. CA Cancer J. Clin. 2007, 57, 278-300. [CrossRef] [PubMed]

15. Khafif, A.; Posen, J.; Yagil, Y.; Beiser, M.; Gil, Z.; Ben-Yosef, R.; Landsberg, R.; Fliss, D.M. Quality of life in patients older than 75 years following major head and neck surgery. Head Neck 2007, 29, 932-939. [CrossRef]

16. Silveira, A.P.; Gonçalves, J.; Sequeira, T.; Ribeiro, C.; Lopes, C.; Monteiro, E.; Pimentel, F.L. Geriatric oncology: Comparing health related quality of life in head and neck cancer patients. Head Neck Oncol. 2011, 3, 3. [CrossRef]

17. Derks, W.; de Leeuw, R.J.; Hordijk, G.J.; Winnubst, J.A. Quality of life in elderly patients with head and neck cancer one year after diagnosis. Head Neck 2004, 26, 1045-1052. [CrossRef]

18. Derks, W.; de Leeuw, J.R.; Hordijk, G.J.; Winnubst, J.A. Elderly patients with head and neck cancer: Short-term effects of surgical treatment on quality of life. Clin. Otolaryngol. Allied Sci. 2003, 28, 399-405. [CrossRef]

19. Laraway, D.C.; Lakshmiah, R.; Lowe, D.; Roe, B.; Rogers, S.N. Quality of life in older people with oral cancer. Br. J. Oral Maxillofac. Surg. 2012, 50, 715-720. [CrossRef]

20. Huang, T.-L.; Tsai, W.-L.; Chien, C.-Y.; Lee, T.-F.; Fang, F.-M. Quality of life for head and neck cancer patients treated by combined modality therapy: The therapeutic benefit of technological advances in radiotherapy. Qual. Life Res. 2010, 19, 1243-1254. [CrossRef]

21. Wan, L.S.; Lee, T.-F.; Chien, C.-Y.; Chao, P.-J.; Tsai, W.-L.; Fang, F.-M. Health-related Quality of life in 640 head and neck cancer survivors after radiotherapy using EORTC QLQ-C30 and QLQ-H\&N35 questionnaires. BMC Cancer 2011, 11, 128.

22. So, W.K.W.; Chan, R.J.; Chan, D.N.S.; Hughes, B.G.M.; Chair, S.Y.; Choi, K.C.; Chan, C.W.H. Quality-of-life among head and neck cancer survivors at one year after treatment-A systematic review. Eur. J. Cancer 2012, 48, 2391-2408. [CrossRef]

23. Nolte, S.; Waldmann, A.; Liegl, G.; Petersen, M.A.; Groenvold, M.; Rose, M. Updated EORTC QLQ-C30 general population norm data for Germany. Eur. J. Cancer 2020, 137, 161-170. [CrossRef]

24. Herce Lopez, J.; Rollon Mayordomo, A.; Lozano Rosado, R.; Salazar Fernandez, C.I.; Gallana, S. Quality of life in long-term oral cancer survivors: A comparison with Spanish general population norms. J. Oral Maxillofac. Surg. 2009, 67, 1607-1614. [CrossRef]

25. Hammerlid, E.; Taft, C. Health-related quality of life in long-term head and neck cancer survivors: A comparison with general population norms. Br. J. Cancer 2001, 84, 149-156. [CrossRef] [PubMed]

26. Bower, J.E.; Ganz, P.A.; Desmond, K.A.; Bernaards, C.; Rowland, J.H.; Meyerowitz, B.E.; Belin, T.R. Fatigue in long-term breast carcinoma survivors: A longitudinal investigation. Cancer 2006, 106, 751-758. [CrossRef] [PubMed]

27. Schmidt, M.E.; Hermann, S.; Arndt, V.; Steindorf, K. Prevalence and severity of long-term physical, emotional, and cognitive fatigue across 15 different cancer entities. Cancer Med. 2020, 9, 8053-8061. [CrossRef] [PubMed]

28. Vilaseca, I.; Chen, A.Y.; Backscheider, A.G. Long-term quality of life after total laryngectomy. Head Neck 2006, 28, 313-320. [CrossRef] [PubMed] 
29. Dahlstrom, K.R.; Bell, D.; Hanby, D.; Li, G.; Wang, L.-E.; Wei, Q.; Williams, M.D.; Sturgis, E.M. Socioeconomic characteristics of patients with oropharyngeal carcinoma according to tumor HPV status, patient smoking status, and sexual behavior. Oral Oncol. 2015, 51, 832-838. [CrossRef]

30. Ringash, J.; Fisher, R.; Peters, L.; Trotti, A.; O’Sullivan, B.; Corry, J.; Kenny, L.; Van Den Bogaert, W.; Wratten, C.; Rischin, D. Effect of p16 status on the quality-of-life experience during chemoradiation for locally advanced oropharyngeal cancer: A substudy of randomized trial Trans-Tasman Radiation Oncology Group (TROG) 02.02 (HeadSTART). Int. J. Radiat. Oncol. Biol. Phys. 2017, 97, 678-686. [CrossRef]

31. Broglie, M.A.; Soltermann, A.; Haile, S.R.; Röösli, C.; Huber, G.F.; Schmid, S.; Stoeckli, S.J. Quality of life of oropharyngeal cancer patients with respect to treatment strategy and p16-positivity. Laryngoscope 2013, 123, 164-170. [CrossRef]

32. Maxwell, J.H.; Mehta, V.; Wang, H.; Cunningham, D.; Duvvuri, U.; Kim, S.; Johnson, J.T.; Ferris, R.L. Quality of life in head and neck cancer patients: Impact of HPV and primary treatment modality. Laryngoscope 2014, 124, 1592-1597. [CrossRef] [PubMed]

33. Bernier, J.; Domenge, C.; Ozsahin, M.; Matuszewska, K.; Lefèbvre, J.-L.; Greiner, R.H.; Giralt, J.; Maingon, P.; Rolland, F.; Bolla, M.; et al. Postoperative Irradiation with or without Concomitant Chemotherapy for Locally Advanced Head and Neck Cancer. N. Engl. J. Med. 2004, 350, 1945-1952. [CrossRef]

34. Cooper, J.S.; Pajak, T.F.; Forastiere, A.A.; Jacobs, J.; Campbell, B.H.; Saxman, S.B.; Kish, J.A.; Kim, H.E.; Cmelak, A.J.; Rotman, M.; et al. Postoperative concurrent radiotherapy and chemotherapy for high-risk squamous-cell carcinoma of the head and neck. $N$. Engl. J. Med. 2004, 350, 1937-1944. [CrossRef]

35. Yoshida, E.J.; Luu, M.; David, J.M.; Kim, S.; Mita, A.; Scher, K.; Shiao, S.L.; Tighiouart, M.; Ho, A.S.; Zumsteg, Z.S. Postoperative chemoradiotherapy in patients with head and neck cancer aged 70 or older with positive margins or extranodal extension and the influence of nodal classification. Head Neck 2018, 40, 1228-1236. [CrossRef]

36. Giacalone, N.J.; Qureshi, M.M.; Mak, K.S.; Kirke, D.; Patel, S.A.; Shah, B.A.; Salama, A.R.; Jalisi, S.; Truong, M.T. Adjuvant chemoradiation does not improve survival in elderly patients with high-risk resected head and neck cancer. Laryngoscope 2018, 128, 831-840. [CrossRef]

37. VanderWalde, N.A.; Meyer, A.M.; Deal, A.M.; Layton, J.B.; Liu, H.; Carpenter, W.R.; Weissler, M.C.; Hayes, D.N.; Fleming, M.E.; Chera, B.S. Effectiveness of chemoradiation for head and neck cancer in an older patient population. Int. J. Radiat. Oncol. Biol. Phys. 2014, 89, 30-37. [CrossRef] [PubMed]

38. Logemann, J.A.; Pauloski, B.R.; Rademaker, A.W.; Lazarus, C.L.; Gaziano, J.; Stachowiak, L.; Newman, L.; MacCracken, E.; Santa, D.; Mittal, B. Swallowing disorders in the first year after radiation and chemoradiation. Head Neck 2008, 30, 148-158. [CrossRef] [PubMed]

39. De Graeff, A.; De Leeuw, J.R.J.; Ros, W.J.G.; Hordijk, G.-J.; Blijham, G.H.; Winnubst, J.A.M. Long-Term Quality of Life of Patients with Head and Neck Cancer. Laryngoscope 2000, 110, 98-106. [CrossRef] [PubMed]

40. Scrimger, R.; Kanji, A.; Parliament, M.; Warkentin, H.; Field, C.; Jha, N.; Hanson, J. Correlation between saliva production and quality of life measurements in head and neck cancer patients treated with intensity-modulated radiotherapy. Am. J. Clin. Oncol. 2007, 30, 271-277. [CrossRef]

41. Jensen, K.; Bonde Jensen, A.; Grau, C. The relationship between observer-based toxicity scoring and patient assessed symptom severity after treatment for head and neck cancer. A correlative cross sectional study of the DAHANCA toxicity scoring system and the EORTC quality of life questionnaires. Radiother. Oncol. 2006, 78, 298-305. [CrossRef] [PubMed]

42. Schuurhuizen, C.; Marino, P.; Braamse, A.M.J.; Buffart, L.M.; Joly, F.; Fizazi, K.; Habibian, M.; Boher, J.M.; Soulie, M.; Oudard, S.; et al. Impact of Patient- and Clinician-Reported Cumulative Toxicity on Quality of Life in Patients with Metastatic CastrationNaïve Prostate Cancer. J. Natl. Compr. Canc. Netw. 2018, 16, 1481-1488. [CrossRef] [PubMed]

43. Sprave, T.; Zöller, D.; Stoian, R.; Rühle, A.; Kalckreuth, T.; Haehl, E.; Fahrner, H.; Binder, H.; Grosu, A.L.; Heinemann, F.; et al. App-Controlled Treatment Monitoring and Support for Head and Neck Cancer Patients (APCOT): Protocol for a Prospective Randomized Controlled Trial. JMIR Res. Protoc. 2020, 9, e21693. [CrossRef] [PubMed]

44. El Shafie, R.A.; Weber, D.; Bougatf, N.; Sprave, T.; Oetzel, D.; Huber, P.E.; Debus, J.; Nicolay, N.H. Supportive Care in Radiotherapy Based on a Mobile App: Prospective Multicenter Survey. JMIR Mhealth Uhealth 2018, 6, e10916. [CrossRef]

45. Denis, F.; Lethrosne, C.; Pourel, N.; Molinier, O.; Pointreau, Y.; Domont, J.; Bourgeois, H.; Senellart, H.; Trémolières, P.; Lizée, T.; et al. Randomized Trial Comparing a Web-Mediated Follow-up with Routine Surveillance in Lung Cancer Patients. J. Natl. Cancer Inst. 2017, 109, djx029. [CrossRef]

46. Basch, E.; Deal, A.M.; Dueck, A.C.; Scher, H.I.; Kris, M.G.; Hudis, C.; Schrag, D. Overall Survival Results of a Trial Assessing Patient-Reported Outcomes for Symptom Monitoring During Routine Cancer Treatment. JAMA 2017, 318, 197-198. [CrossRef]

47. Hofstede, G. The cultural relativity of the quality of life concept. Acad. Manag. Rev. 1984, 9, 389-398. [CrossRef]

48. Morton, R.P. Studies in the quality of life of head and neck cancer patients: Results of a two-year longitudinal study and a comparative cross-sectional cross-cultural survey. Laryngoscope 2003, 113, 1091-1103. [CrossRef] [PubMed]

49. Haehl, E.; Ruhle, A.; David, H.; Kalckreuth, T.; Sprave, T.; Stoian, R.; Becker, C.; Knopf, A.; Grosu, A.L.; Nicolay, N.H. Radiotherapy for geriatric head-and-neck cancer patients: What is the value of standard treatment in the elderly? Radiat. Oncol. 2020, 15, 31. [CrossRef]

50. Sprave, T.; Rühle, A.; Stoian, R.; Weber, A.; Zamboglou, C.; Nieder, C.; Grosu, A.-L.; Nicolay, N.H. Radiotherapy for nonagenarians: The value of biological versus chronological age. Radiat. Oncol. 2020, 15, 113. [CrossRef] [PubMed]

51. Schmidt, J.; Lamprecht, F.; Wittmann, W. Satisfaction with inpatient management. Development of a questionnaire and initial validity studies. Psychother. Psychosom. Med. Psychol. 1989, 39, 248-255. [PubMed] 